\title{
Rickettsiosis: A Case Series on Different Clinical Presentation in Children
}

\author{
Maria Candelino, Paola Di Filippo*, Debora Mariarita d’Angelo, Daniela David, Francesco Sansone, \\ Carmela Salladini, Rita Greco, Nadia Rossi and Francesco Chiarelli \\ Department of Pediatrics, University of Chieti-Pescara, Italy
}

*Corresponding author: Paola Di Filippo, Department of Pediatrics, University of Chieti, Italy.

To Cite This Article: Paola Di Filippo, Rickettsiosis: A Case Series on Different Clinical Presentation in Children. 2020 - 9(4). AJBSR.MS.ID.001401. DOI: 10.34297/AJBSR.2020.09.001401.

Received: 眥: June 16, 2020; Published: 䇦 July 06, 2020

\begin{abstract}
Mediterranean spotted fever (MSF) is an acute febrile, zoonotic disease caused by Rickettsia conorii. This is transmitted to humans by the brown dogtick Rhipicephalus sanguineus. Although therapy is easy and unexpensive, the different clinical presentation, the lack tick bite in the history or the frequent absence of skin manifestations, often make diagnosis difficult. We here reported four clinical cases of MSF, occurred in a region of central Italy (Abruzzo) with different clinical characteristics, but common epidemiological and anamnestic characteristics.
\end{abstract}

Keywords: Raoult Score; Rickettsia Conorii; Tick Bite; Tache Noir

Abbreviations: MFS: Mediterranean Spotted Fever; Nv: Normal Value; CRP: C-Reactive Protein; ERS: Erythrocyte Sedimentation Rate; EBV: Ebstein-Barr Virus

\section{Introduction}

Mediterranean spotted fever (MSF) is a tick-borne acute febrile disease caused by Rickettsia conorii. This pathogen is transmitted to humans by the brown dog tick Rhipicephalus sanguineus, which is both the vector and reservoir of infection and it is endemic in Mediterranean countries and North Africa [1]. Besides, recently others rickettsiosis pathogens have been associated with human diseases (R. slovaca, R. aeschlimanni, R. massilliae, R. monacensis, R. conorii Israelensis) in Europe, and they have been identified in various arthropod hosts, which can parasitize some vertebrates, including the dog $[2,3]$.

Spotted fever group rickettsiosis are re-emerging agents of tickborne diseases in European countries of Mediterranean area. In Italy, especially in Southern (Sardinia, Sicily, Calabria) and Central regions, about 1000 cases/year are reported, with an annual hospitalization rate of $1,36 / 100.000$ persons year. About $20 \%$ of cases are reported in pediatric age with a seasonal pattern from April to September [2]. MSF is mostly characterized by flu-like symptoms, maculopapular rash and a black eschar at the site of the tick bite ('tache noir'). Typical symptoms are rarely present at the same time, especially in children, representing a diagnostic challenge.
Early antibiotic treatment, moreover, shortens the symptomatic period of MSF infection and it prevents the occurrence of severe complications [4]. Currently, given the better tolerance and the equal clinical response compared to chloramphenicol and tetracyclines, the macrolides represent the first line treatment, especially in pediatric age [5]. Four clinical cases of MSF are here presented. They occurred in a region of central Italy (Abruzzo), with different clinical presentation, but common epidemiological and anamnestic characteristics.

\section{Case 1}

A 10-year-old Caucasian boy presented an intermittent bi-quotidian fever up to $39.7^{\circ} \mathrm{C}$, associated to asthenia and sore throat. Cough, skin rashes, arthralgia or other symptoms were not referred. Because of the Coronavirus 19 pandemic, in the previous 60 days the family was in domiciliary quarantine in a small town in Abruzzo, living in a rural area near a farm guesting several animals. Antibiotic therapy with azithromycin for two days and after amoxicillin/clavulanic acid for six days was administered with no defervescence. Therefore, the child was conducted to the Emergency Room after 15 fever days. Blood tests revealed increased CRP 
(110,4 mg/L, nv<5 mg/L), erythrocyte sedimentation rate (ESR: 49 $\mathrm{mm} / \mathrm{h}, \mathrm{nv}<20 \mathrm{~mm} / \mathrm{h})$ and D-dimer $(2,45 \mathrm{mg} / \mathrm{L})$, and a neutropenia $(880 / \mathrm{uL})$.

Chest X-ray was normal. An intravenous empiric broad- spectrum antibiotic treatment with ceftriaxone was started. Blood, urine and stool culture were negative. An electrocardiogram and echocardiogram ruled out an endocarditis and a valvular involvement. In addition, abdomen ultrasounds did not find hepatomegaly, splenomegaly or abdominal abscesses. The rhinopharyngeal swab and serologic tests for SARS-CoV2 were performed twice and resulted negative. The research for Bartonella, Brucella, Borrelia, Toxoplasma, Leishmania, Mycoplasma, Chlamydia, CMV, EBV, Adenovirus and Mycobacterium tuberculosis was negative. A bone marrow aspiration showed a reactive inflammatory pattern. The autoimmune antibodies panel (ANA, ENA, rheumatoid factor test) was negative. In consideration of the fever persistence, antibiotic therapy with Clarithromycin was added. After 48 hours, the patient presented the last feverish peak, remaining afebrile thereafter. Serological test for Rickettsia conorii resulted positive (IgM title 1:40, IgG title 1:80 and total Ig title 1:40; convalescent serum: IgM title 1:80 and IgG title 1:80; total Ig: 1: 90).

\section{Case 2}

A 15 years-old Caucasian boy presented to our Pediatric Department in summertime with intermittent fever starting three day earlier, associated to dry cough and frontal headache.

The physical examination showed only two little scabs in the thighs root. In the recent past history, he referred about a mountain trip two weeks earlier and about two little tick bite ticks rapidly removed, leaving only an erythematous halo at the puncture site for few days. Laboratory tests revealed only an increased CRP (5,72 mg/dl; nv <0,5 mg/dl). Chest X-ray, echocardiogram and abdomen ultrasounds were normal. It was started an oral antibiotic treatment with amoxicillin/clavulanic acid. Serology for EBV, Borrelia Burgdorferi, Adenovirus, Mycoplasma and Chlamydia resulted negative for acute infection. During the hospitalization, a thrombocytopenia $(68.000 / \mu \mathrm{l})$ was developed: the antibodies-antiplatelet were negative, a pediatric hematologist hypothesized a thrombocytopenia related to an infection event. Serology for Rickettsia conorii with the immunofluorescent assay resulted positive (IgM title 1:80, IgG title 1:130, total IgG title 1:140). An oral treatment with Azithromycin for 5 days was started with consequent defervescence after 4 total days of fever.

\section{Case 3}

A 14-years-old Caucasian boy presented to our Pediatric Department during springtime with bitemporal headache, an episode of vomiting followed by spatiotemporal disorientation. The physical examination showed normal neurologic assessment and two little bites on the left thigh. The boy experienced a mountain trip 10 days before and he reported that he wore shorts. He did not refer any bite. Laboratory tests resulted normal. Chest X-ray and echocardiogram were normal. An electroencephalography revealed a pattern indicative for a encephalitic involvement. To rule out a meningeal/ encephalitic process, a head CT, head MRI and a lumbar puncture were performed and resulted normal. Serology for Borrelia Burgdorferi, Mycoplasma, Chlamydia and SARS-CoV-2 resulted negative. Autoimmune parameters resulted negative. A therapy with cephalosporin antibiotics and antiviral, plus steroid therapy was started. The immunofluorescent assay for Rickettsia conorii resulted positive (IgM title 1:40, IgG title 1:40, Ig total title 1:40, convalescent serum: IgM title 1:40 and IgG title 1:80; total IgG: 1: 80). A therapy with macrolides (oral Azithromycin), for 10 days was started with a gradual resolution of symptoms and the electroencephalography documented a normalization of the voltage for the patient's age.

\section{Case 4}

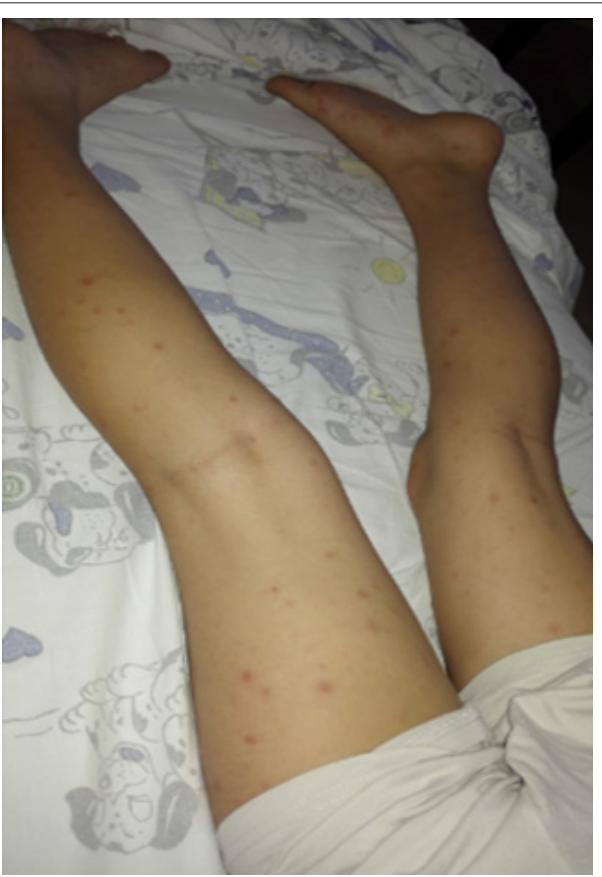

Figure 1: The macular rash of the legs with involvement of the soles of the feet presented by the case 4 .

A 7 years old Caucasian boy presented to our Pediatric Department in summertime for a 6-days history of continuous fever, associated to arthralgias and myalgias of the lower limbs. The physical examination revealed a macular rash of the legs with involvement of the soles (Figure 1). A pustular small not itchy lesion $(0.3 \times 0.2 \mathrm{~cm})$ with an erythematous contour at the right auricle, associated to a painful reactive lymphadenitis in ipsilateral lateral cervical region was also found. No trauma, tick or insect bites were reported at the site of skin lesion. Laboratory tests showed only a slight increase in the inflammation indices (CRP: 1,46 mg/dl, $\mathrm{nv}<0,5 \mathrm{mg} / \mathrm{dl}$; ESR: $35 \mathrm{~mm} / \mathrm{h}$, nv $5 \mathrm{~mm} / \mathrm{h}$ ). Therapy with ceftriaxone was started, but fever continued. Serology for Adenovirus, CMV, Coxsackie, Parvovirus B19, Mycoplasma, Chlamydia and EBV were negative for acute 
infection. Stool, urine, blood and throat cultures were negative. The peripheral blood smear test and the autoimmune antibodies panel resulted no pathologic. On the fifth day of hospitalization a spontaneous drainage of the pustular auricular lesion was reported, with an evolution in ulcer with central eschar (Figure 2). The grandfather reported that the boy played often with his dog a week before the onset of the fever. Therefore, in suspicion of MSF, a therapy with clarithromycin was started, with prompt defervescence and gradual disappearance of joint pain. Indirect immunofluorescent antibody assay for Rickettsia conorii confirmed the clinical suspicion (IgG: 1: 1:40, total Ig: title 1:80; convalescent serum: IgM title 1:80 and IgG title 1:80; total IgG: 1: 120).

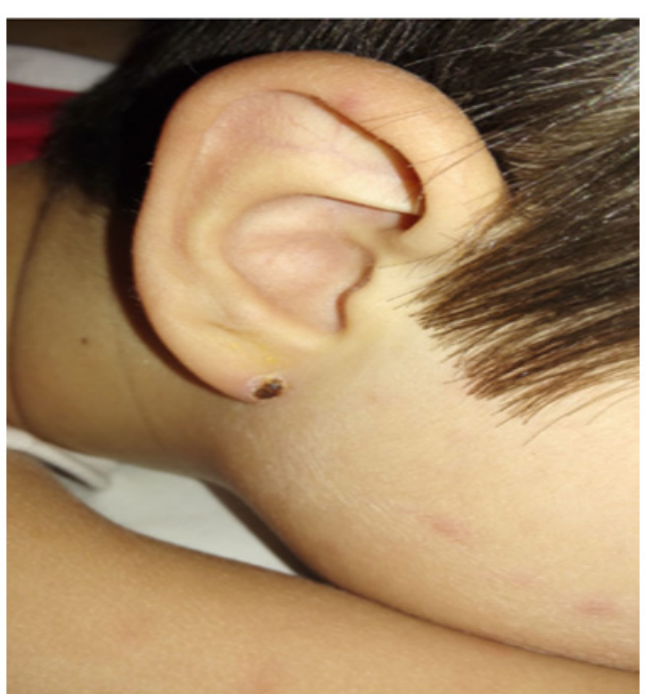

Figure 2: Tache noir presented by the case 4 .

\section{Discussion}

Rickettsioses are worldwide zoonoses, nowadays re-emerging and changing in distribution in several regions of the world [4]. Rickettsia are a gram- negative, non-spore-forming and obligate intracellular parasites which can use both vertebrate and invertebrate as hosts and as primary reservoirs and vectors [6]. Therefore, a contact with dogs is not necessary and in a large Italian case series this risk factor was reported only by $20.7 \%$ of patients [4]. Similarly, only one of our patients reported a prolonged contact with a dog in his history. Recently Pasucci et al. [3] analyzed 603 ticks in Central Italy (Abruzzo and Molise) finding a high rate of Rickettsia infection (50,2\%) and 8 different species of Rickettsia, including Rickettsia conorii sp. conorii, which is the most frequent responsible for MSF.3 Generally, after an incubation period between 5 and 10 days after the bite of the infected tick, MSF has a sudden onset, with flu-like symptoms (moderate or high fever accompanied by chills, asthenia, headache, general disorders). The maculo-papular exanthema appears on the 3rd-5th day of illness, first in the wrists and ankles, then spreads to the palms and soles, until it affects the trunk and rarely the face [7]. This is the symptom of a vasculitis related to the infection [8] In pediatric cases, instead, fever and rash are the most common symptoms (about $90 \%$ of cases).

Three out of four cases here presented, conversely, were without rash. Non-exanthematic forms can partially explain the discrepancy between the high prevalence of seropositivity and the prevalence of the infection reported in some studies [7] Only for one of our cases it was possible to find the typical eschar and this factor significantly improve the diagnosis. Moreover, the typical eschar is found in $40-60 \%$ of pediatric patients $[4,8,9]$ and the most common localizations are the scalp, the neck, and the truncus [7]. The infectious tick bite is painless and often goes unnoticed. A history of tick bite is an important data of the history of the patient, but it is often absent, making diagnosis more difficult.7 Most of our patients were unaware of tick bites, in line with the data in the literature. The non-specific presentation of Rickettsia infection and the absence of typical signs often make differential diagnosis difficult, especially because the history of the tick bite is missing. Symptoms occur mildly in $80 \%$ of cases. In presence of peculiar conditions (diabetes, glucose 6 phosphate dehydrogenase deficiency, cirrhosis...) the clinical course can be severe and also fatal (malignant MSF, 1-7 \% of cases), because of complications like acute renal failure, thrombocytopenia, myocarditis, pneumonitis, gastric hemorrhage, shock, and even multiple organ failure [10,11]. Involvement of the central nervous system, which has been most frequently reported in adulthood, is extremely rare in children [11,12]. Headache is complained more by adults compared to children, contributing to a milder clinical presentation compared to adults $[4,13]$. One of our patients, however, developed severe neurological symptoms. In spite of this clinical presentation variability, some epidemiological-anamnestic features are almost the same and they can guide the clinical suspicion: males are more affected than females; the infection occurs mainly during spring and summer, and mostly in people who live in rural or wooded areas, because of a higher exposure to ticks $[2,4,14,15]$ About laboratory characteristics, acute phase reactants are mildly increased; 4 all our patients experienced increased inflammatory indices. Leucopenia with an alteration of neutrophils is observed in $25-50 \%$ of patient; 4 in fact, two of our patients presented relative neutropenia. Thrombocytopenia and anemia might also be present 4 and one patient presented normochromic normocytic anemia and another one developed thrombocytopenia Hypertransaminasaemia and alteration of coagulation tests are other non-specific common findings.4 Microscopic hematuria with or without mild proteinuria or albuminuria is also observed because of a glomerular involvement $[4,8]$.

The indirect immunofluorescent antibody (IFA) test is the current serological gold standard and reference test [1]. All these clinical, epidemiological and laboratory aspects are summarized in the Raoult [16] score, used to assess the possibility of Rickettsia infection [16]. In all of our patients indeed, despite the atypical presentation, the score was > 25 (table 1). The American Academy of Pediatrics did not recommend tetracyclines for infections in children 
younger than eight years of age. Macrolides seems to have similar efficacy of doxycycline and chloramphenicol and a significantly better tolerability, especially in children [5,17] Given the milder clinical presentation and the benign evolution of MSF, macrolides (effective against Rickettsia conorii) can be safety used in children [4]. All the patients were successfully treated with oral macrolides, in line with the recent literature data. In conclusion, in presence of acute febrile illness in children, especially in the spring-summer period, it is mandatory to exclude the Rickettsia infection. Luckily, the clinical evolution in children is benign, but sometimes it can be severe. Early treatment can improve the outcome of the child, also shortening the time of defervescence.

Table 1: Diagnostic Raoult score for MSF, calculated for all the four presented patients. A total $>25$ is consistent with a diagnosis of MSF.0

\begin{tabular}{|c|c|c|c|c|c|}
\hline Criteria & Score & Case 1 & Case 2 & Case 3 & Case 4 \\
\hline \multicolumn{6}{|c|}{ Epidemiologic Criteria } \\
\hline Life or recent travel in endemic area & 2 & 2 & 2 & 2 & 2 \\
\hline Onset between May and September & 2 & 2 & 2 & 2 & 2 \\
\hline Contact with dog's ticks & 2 & & & & \\
\hline \multicolumn{6}{|l|}{ Clinical Criteria } \\
\hline Fever higher than $39^{\circ} \mathrm{C}$ & 5 & 5 & 5 & 5 & 5 \\
\hline Eschar "Tache noire" & 5 & & & & 5 \\
\hline Maculopapular or purpuric eruption & 5 & & & & \\
\hline Two out of these three clinical criteria & 3 & & & & 3 \\
\hline All the three clinical criteria together & 5 & & & & \\
\hline \multicolumn{6}{|c|}{ Unspecific Biological Criteria } \\
\hline Platelet count $<150000 / \mathrm{mm}^{3}$ & 1 & & 1 & & \\
\hline Liver enzyme > $50 \mathrm{IU} / \mathrm{L}$ & 1 & & & & \\
\hline \multicolumn{6}{|c|}{ Bacteriological Criteria } \\
\hline Isolation of Rickettsia conorii from blood & 25 & & & & \\
\hline Detection of R. conorii in skin biopsy using IFA & 25 & & & & \\
\hline \multicolumn{6}{|c|}{ Serological Criteria (Immunofluorescence) } \\
\hline Sole serum with total lg le 1:128 & 5 & & 5 & & \\
\hline Sole serum with $\operatorname{IgG}>1: 128$ and $\operatorname{IgM}>1: 64$ & 10 & & 10 & & \\
\hline 4-fold increase in titer in a double serum sample within 2 weeks & 20 & 20 & & 20 & 20 \\
\hline Total & & 29 & 25 & 29 & 47 \\
\hline
\end{tabular}

\section{Reference}

1. Figoni J, Chirouze C, Hansmann Y (2019) Lyme borreliosis and other tick-borne diseases. Guidelines from the French Scientific Societies (I) prevention, epidemiology, diagnosis. Med Mal Infect 49(5): 318-334.

2. Gomez Barroso D, Vescio MF, Bella (2019) A Mediterranean spotted fever rickettsiosis in Italy, 2001-2015: Spatio-temporal distribution based on hospitalization records. Ticks Tick Borne Dis 10(1): 43-50.

3. Pascucci I, Di Domenico M, Curini V (2019) Diversity of Rickettsia in Ticks Collected in Abruzzi and Molise Regions (Central Italy). Microorganisms 7(12): 696 .

4. Colomba C, Saporito L, Polara VF (2006) Mediterranean spotted fever: clinical and laboratory characteristics of 415 Sicilian children. BMC Infect Dis 6: 60.

5. Anton E, Muñoz T, Travería FJ, Navarro G (2016) Randomized trial of clarithromycin for Mediterranean spotted fever. Antimicrob Agents Chemother 60(3): 1642-1645.

6. lano JP (2005) Rickettsial infections. Ann N Y Acad Sci 1063: 187-196.

7. Cascio A, Iaria C (2006) Epidemiology and clinical features of Mediterranean Spotted Fever in Italy. Parassitologia 48(1): 131-133.
8. Galanakis E, Bitsori M (2011) Rickettsioses in children: a clinical approach. Adv Exp Med Biol 719: 145-162.

9. Buckingham SC, Marshall GS, Schutze GE (2007) Tick-borne Infections in Children Study Group. Clinical and laboratory features, hospital course, and outcome of Rocky Mountain spotted fever in children. J Pediatr 150: 180-184.

10. Herath, HMLY, Jayasundara, JMHD, Senadhira SDN (2018) Spotted fever rickettsioses causing myocarditis and ARDS: a case from Sri Lanka. BMC Infect Dis 18(1): 705

11. Baltadzhiev I, Popivanova N, Zaprianov Z (2016) Malignant forms of Mediterranean spotted fever: risk factors for fatal outcomes. Braz J Infect Dis 20(5): 511-512.

12. Sekeyová Z, Danchenko M, Filipčík P (2019) Rickettsial infections of the central nervous system. Plos Negl Trop Dis 13(8): 0007469.

13. Abdad MY, Abou Abdallah R, Fournier PE (2018) A Concise Review of the Epidemiology and Diagnostics of Rickettsioses: Rickettsia and Orientia spp. J Clin Microbiol 56(8): 01728-17.

14. (2003) American Academy of Pediatrics. Prevention of tick-borne infections. In: Pickering LK, editor. 2003 Red Book: Report of the Committee on Infectious Diseases. 26th ed. Elk Grove Village (IL): American Academy of Pediatrics: $186-7$ 
15. Parola P, Paddock CD, Socolovschi C (2013) Update on tick-borne rickettsioses around the world: a geographic approach. Clin Microbiol Rev 26: 657-702.

16. Raoult D, Tissot-Dupont H, Caraco P, et al. (1992) Mediterranean spotted fever in Marseille: descriptive epidemiology and the influence of climatic factors. Eur J Epidemiol 8(2): 192-197.
17. Cascio A, Colomba C, Di Rosa D (2001) Efficacy and Safety of Clarithromycin as Treatment for Mediterranean Spotted Fever in Children: A Randomized Controlled Trial. Clinical Infectious Diseases 33(3): 409-411. 